\title{
The Role of Urinary Biomarkers as Diagnostic and Prognostic Predictors of Acute Kidney Injury Associated With Vancomycin
}

\section{OPEN ACCESS}

Edited by:

Marlies Ostermann

Guy's and St Thomas' NHS

Foundation Trust, United Kingdom

Reviewed by:

Ryan Williams,

City College of New York (CUNY),

United States

Brijesh Yadav,

Sanjay Gandhi Post Graduate Institute

of Medical Sciences (SGPGI), India

*Correspondence: Daniela Ponce daniela.ponce@unesp.br

Specialty section:

This article was submitted to

Renal Pharmacology,

a section of the journal

Frontiers in Pharmacology

Received: 05 May 2021

Accepted: 30 July 2021

Published: 23 September 2021

Citation:

Sampaio de Souza Garms D, Cardoso Eid KZ, Burdmann EA, Marçal LJ, Antonângelo L, Santos Ad and Ponce D (2021) The Role of Urinary Biomarkers as Diagnostic and Prognostic Predictors of Acute Kidney Injury Associated With Vancomycin.

Front. Pharmacol. 12:705636.

doi: 10.3389/fphar.2021.705636
Durval Sampaio de Souza Garms ${ }^{1}$, Karina Zanchetta Cardoso Eid ${ }^{1}$, Emmanuel A. Burdmann ${ }^{2}$, Lia Junqueira Marçal ${ }^{1}$, Leila Antonângelo ${ }^{2}$, Adriano dos Santos ${ }^{3}$ and Daniela Ponce ${ }^{1 *}$

${ }^{1}$ Department of Internal Medicine, University of São Paulo State, São Paulo, Brazil, ${ }^{2}$ LIM 12, Nephrology Discipline of University of São Paulo, São Paulo, Brazil, ${ }^{3}$ Clinics Hospital Pharmacy, Botucatu School of Medicine, São Paulo, Brazil

Introduction: The incidence of acute kidney injury (AKI) related to vancomycin is variable, and several risk factors related to the treatment and patients may explain the nephrotoxicity. The role of urinary biomarkers in AKI related to vancomycin is unknown.

Objective: The aim of this study was to evaluate the role of urinary IL-18, KIM-1, NGAL, TIMP2, and IGFBP7 as diagnostic and prognostic predictors of AKI related to vancomycin.

Methods: A prospective cohort study of patients receiving vancomycin and admitted to wards of a public university hospital from July 2019 to May 2020 was performed. We excluded patients that had AKI before starting vancomycin, hemodynamic instability, inability to collect urine, and chronic kidney disease stage 5.

Results: Ninety-four patients were included, and the prevalence of AKI was $24.5 \%$, while the general mortality was $8.7 \%$. AKl occurred $11 \pm 2$ days after the first vancomycin dose. The most frequent KDIGO stage was 1 (61\%). There was no difference between patients who developed and did not develop AKI due to gender, length of hospital stay, dose, and time of vancomycin use. Logistic regression identified age (OR 6.6, Cl 1.16-38.22, $p=0.03$ ), plasmatic vancomycin concentrations between 96 and $144 \mathrm{~h}$ (OR 1.18, $\mathrm{Cl} 1.04-1.40, p=0.04)$, and urinary NGAL levels between 96 and $144 \mathrm{~h}$ (OR 1.123, Cl 1.096-1.290, $p=0.03$ ) as predictors of AKI. The time of vancomycin use (OR 4.61, $\mathrm{Cl} 1.11-22.02, p=0.03$ ), higher plasmatic vancomycin concentrations between 192 and $240 \mathrm{~h}$ (OR 1.02, Cl 0.98-1.06, $p=0.26$ ), and higher cell cycle arrest urinary biomarkers TIMP-2 multiplied by IGFBP-7 between 144 and $192 \mathrm{~h}$ (OR 1.33, Cl 1.10-1.62, $p=0.02$; OR 1.19, $\mathrm{Cl} 1.09-1.39, p=0.04$, respectively) were identified as prognostic factors for non-recovery of kidney function at discharge.

Conclusion: AKI related to vancomycin was frequent in patients hospitalized in wards. Age, plasmatic vancomycin concentrations, and NGAL between 96 and $144 \mathrm{~h}$ were identified as predictors of AKI related to vancomycin use. Plasmatic vancomycin concentrations and urinary NGAL were predictors of AKI diagnosis within the next 5 days. The urinary biomarkers of cell cycle arrest TIMP-2 and IGFBP-7 and the duration of vancomycin use were associated with non-recovery of kidney function at hospital discharge moment.

Keywords: acute kidney injury, nephrotoxicity, vancomycin, biomarkers, IL-18, NGAL, IGFBP7, TIMPS-2 


\section{INTRODUCTION}

Vancomycin is a widely used drug in intensive care units (ICUs), as oxacillin-resistant gram-positive cocci are the main infectious etiological agents. The main polemic points on vancomycin relate to its efficacy and safety. Regarding efficacy, the concern is the alteration in pharmacokinetics, usually related to the distribution, metabolism, and elimination of the drug, which can lead to subtherapeutic concentrations, leading to the emergence of bacterial resistance due to the inefficacy of the treatment and consequently leading to higher mortality. Regarding safety, emphasis has been given to the main side effect of the medication-nephrotoxicity - which can aggravate the clinical picture and contribute to unfavorable outcomes and occurs 4-8 days after starting to use. The pathophysiological mechanisms are not yet fully understood, but undoubtedly it is ongoing with a consequent worsening of the prognosis of these patients (Zamoner et al., 2020).

The incidence of vancomycin nephrotoxicity varies widely between different studies, reaching up to $40 \%$ and there are different factors related to the patient and/or the drug that can accelerate the nephrotoxicity. Among the factors associated with the patient, we highlight, mainly, advanced age, sepsis, dehydration, and reduced kidney function, while among the risk factors related to the drug are the concomitant administration with other drugs such as loop diuretics, amphotericin B, aminoglycosides, vasopressors, and intravenous contrast media; the longer duration of treatment and the high plasmatic vancomycin concentration (Elyasi et al., 2012; Zamoner et al., 2020).

It is recommended that the monitoring of the plasmatic concentration of vancomycin be performed in patients at high risk for renal dysfunction, critically ill, in those who receive high vancomycin doses and/or in treatment for more than 5 days.

Some authors, however, still question whether the high plasmatic vancomycin concentrations are the cause or the consequence of AKI (Elyasi et al., 2012; Hanrahan et al., 2014).

Changes in plasmatic creatinine and urine output are still the standards for recognizing AKI. However, it is known that creatinine and urine output are influenced by factors such as hypercatabolism, drugs, hydration status, muscle mass, gender, and age, which can delay the diagnosis of AKI. In addition, AKI may be present even in the absence of an increase in plasma creatinine due to renal functional reserve or tubular creatinine secretion. These limitations for the use of serum creatinine spurred research to discover new, more sensitive, specific, and early biomarkers in the diagnosis and prognosis of AKI, including the tubular lesion markers kidney injury molecule-1 (KIM-1), interleukin 18 (IL-18), neutrophil gelatinase-associated lipocalin (NGAL), tissue inhibitor of metalloproteinases 2 (TIMP-2), and insulin-like growth factor binding protein 7 (IGFBP-7) (Andreucci et al., 2017; Schrezenmeier et al., 2017).

Today there is no perfect biomarker of AKI. Each biomarker is not completely specific for AKI. This is reflected by the imperfect test characteristics of each biomarker with the best AUCROCs ranging between 0.75 and 0.85 . The pathophysiologic basis for this lack of sensitivity and specificity is only partially understood.
For instance, NGAL and IL-18 are known to be produced in immune cells and display associations with critically ill patients and sepsis, which are independent of AKI. NGAL, KIM-1, and IL18 are elevated in patients with CKD. TIMP-2 and IGFBP7 have not been thoroughly tested in settings outside the ICU, and their pathophysiological roles are currently unclear. NGAL is closely associated with intrinsic AKI and displays much lower levels in pre-renal AKI, which makes them potentially suitable in the differential diagnosis of patients with established AKI (Andreucci et al., 2017; Schrezenmeier et al., 2017).

Few studies have been carried out to predict AKI using biomarkers in the context of nephrotoxicity due to the use of antibiotics. This study aimed to evaluate the role of urinary biomarkers IL-18, KIM-1, NGAL, TIMP-2, and IGFBP7 as diagnostic and prognostic predictors of AKI associated with vancomycin in hospitalized patients.

\section{METHODS}

\section{Study Population}

This was a cohort study designed to analyze the development of AKI related to vancomycin use. It included patients 18 years of age or older admitted to the wards of Clinical Hospital from Botucatu School of Medicine and that received vancomycin. These patients were followed from the time of admission to wards until wards discharge. Nephrology fellows visited the wards daily from July 2019 to May 2020 and collected data on all patients admitted.

Exclusion criteria were chronic kidney disease (CKD) patients stages 4 and 5 , kidney transplantation, wards stay $<48 \mathrm{~h}$, patients in the process of installing or recovering AKI of any etiology prior to the start of vancomycin, critical patients with a shock of any etiology and/or need for vasopressors, patients who were receiving others nephrotoxicity drugs as amikacin and amphotericin B. and disability in the collection of urinary samples.

Baseline glomerular filtration rate was estimated using the clearance de creatinine estimated by the CKD EPI equation (Levey et al., 2009). Baseline creatinine was defined as the lowest serum creatinine value in the last 6 months before AKI or, for those without this measurement, the lowest value achieved during hospitalization in the absence of dialysis (Siew et al., 2010). Recovery of kidney function was defined as the return of creatinine levels to baseline value at discharge hospital moment. Figure 1 shows the inclusion and exclusion criteria.

Patient data were collected by the same observer, through consultations with the patient and their medical records, from the beginning of vancomycin until its outcome (discharge or death). Clinical (gender, age, weight, comorbidities, infectious focus, diuresis), laboratory (baseline creatinine), and vancomycin (doses, days of use, plasmatic concentrations) data were collected daily. AKI was defined and classified according to KDIGO criteria (KDIGO Acute Kidney Injury Work Group, 2012), and toxic serum vancomycin concentrations were defined as trough value higher than 20 mg/L (Rybak et al., 2009). 
218 patients in vancomycin use in wards during 2019

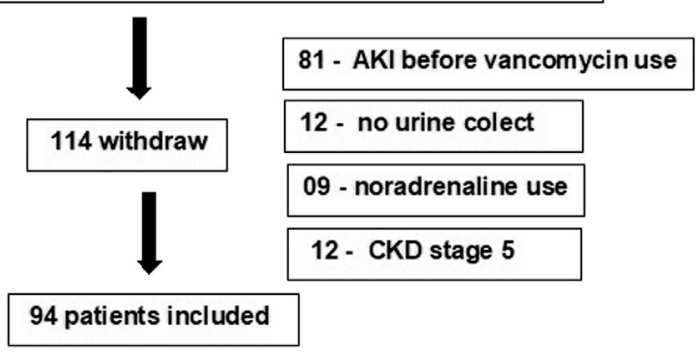

FIGURE 1 | Patients undergoing vancomycin and hospitalized in wards and included in the study.

Dosages of uIL-18, uKIM-1, uNGAL, TIMP-2, and uIGFBP7 and plasmatic vancomycin concentrations were performed every 48-72 h from the first day of vancomycin use until the end of antibiotic therapy. All values were normalized for urinary creatinine.

This study was sent to the institution's Research Ethics Committee for analysis and started only after its approval (protocol 2,931,887).

\section{Biochemical Analysis}

The urinary samples were centrifuged at 2,000 rpm for $5 \mathrm{~min}$ and stored at minus 80F0B0C and were analyzed subsequently.

Urinary IL-18, KIM-1, NGAL, TIMP-2, and IGFBP7 were measured using LUMINEX-xMAP technology at the University of Sao Paulo with reading on a MagPix device ((MerckMillipore, Austin, Texas, United States), using three kits:

- Human renal toxicity Bio-Plex Pro RBM PANEL 1 (Bio-Rad Laboratories, Berkeley, California, United States): IL-18 and KIM-1

- Human renal toxicity Bio-Plex Pro RBM PANEL 2 (Bio-Rad Laboratories, Berkeley, California, United States): NGAL

- MILLIPLEX $^{\circledR}$ MAP Custom Human 2-plex Magnetic Bead panel (EMD MerckMillipore, Saint Louis, Missouri, United States): TIMP-2 and IGFBP-7

Urine samples from six patients were repeated in quintuplicate to assess the reproducibility of the assays. The coefficient of variation within and between assays was 15 and 20\%, respectively.

The NephroCheck ${ }^{\circledR}$ kit is a rapid test in which the TIMP-2 concentration is measured multiplied by the IGFBP-7 value, and the result is divided by 1,000 . As above $0.3(\mathrm{ng} / \mathrm{ml}) / 1,000$ are positive for the test.

In addition to the dosages of BM options, we also analyzed the performance of the markers that make up the NephroCheck ${ }^{\circledR}$ kit and their relationship.

\section{Statistical Analysis}

Data analysis was performed using IBM SPSS Statistics 24.0 (United States, 2018). Continuous variables with non-normal distribution were described using median and interquartile range and those with a normal distribution as means \pm standard deviation. Categorical variables were presented as $n(\%)$. Student's t-test was used for data with a parametric distribution and the KruskalWallis test for non-normal data for the analysis of continuous variables. For the analysis of categorical variables, a chi-square test was used.

Diagnostic characteristics of vancomycin concentrations and urinary NGAL in predicting AKI and TIMP-2 and IGFBP-7 in predicting recovery of kidney function were assessed by the calculation of the area under the receiver operating characteristic curve (AUCROC). AUCROC analysis was performed by comparing AKI patients with all non-AKI patients and by comparing patients who recovered kidney function at hospital discharge moment with those that did not recover it. In all tests, differences were considered significant at $5 \%$.

\section{RESULTS}

Ninety-four patients were included in the final analysis (Figure 1). AKI incidence was 24.5\%, and it occurred $11 \pm$ 3 days after the start of vancomycin. The mortality rate was $8.7 \%$. Mean age was $51.1 \pm 15.5$ years, $67 \%$ were male, most of them had comorbidities $(63.8 \%)$, and hypertension was the most frequent (in $47.9 \%$ ) One hundred fourteen patients were excluded mainly due to present AKI before the introduction of vancomycin (81 patients) or due to difficulty in urine collection (Bagshaw et al., 2010) or need for noradrenalin (09 patients) or CKD stage 4 or 5 . Ninety-one patients (96.8\%) had vancomycin concentrations measured sequentially, and all had at least one creatinine measured during the use of the antibiotic.

Among AKI patients (23 patients), AKI KDIGO 1 occurred in 14 patients (61\%). The two groups (AKI vs non-AKI) were similar in gender, length of stay, dose, and time of vancomycin use. Patients who developed AKI were older $(p=$ $0.01)$, infection was the main cause of hospitalization $(p=0.02)$, prevalence of hypertension was higher $(p=0.001)$, plasmatic creatinine at admission was higher $(p=0.001)$, and vancomycin concentrations among 48-96 h $(p=0.006), 96-144 \mathrm{~h}(p=0.037)$, $144-192 \mathrm{~h}(p=0.003)$, and 192-240 h $(p=0.016)$ were higher, as shown in Table 1.

AKI patients had higher levels of urinary IL-18 between 144 and $192 \mathrm{~h}(p=0.027)$, KIM- 1 between 96 and $144 \mathrm{~h}(p=0.005)$, NGAL between 96 and $144 \mathrm{~h}$ and 144-192 h $(p=0.04, p=$ $0.031, p=0.012$, respectively), as shown in Table 2. After normalizing the biomarkers values for urinary creatinine, there was no difference in KIM-1 levels, and AKI patients kept higher levels of urinary IL-18/creatinine between 144 and $192 \mathrm{~h}(p=0,03)$ and urinary NGAL/creatinine between 96$144 \mathrm{~h}$ and $144-192 \mathrm{~h}$ ( $p=0.05$ and $p=0.008$, respectively) as shown in Table 3. Age (OR 6.6 CI 1.16-38.22, $p=0.03$ ), vancomycin concentrations, and urinary NGAL between 96 and $144 \mathrm{~h}$ (OR $1.18 \mathrm{CI} 1.04-1,40 p=0.04$; OR 1.123, CI $1.096-1.290, p=0.03$, respectively) were identified as predictors of AKI at logistic regression (Table 4). 
TABLE 1 | Patients demographics and clinical characteristics during hopitalization at wards.

\begin{tabular}{|c|c|c|c|}
\hline & Non-AKI $n=71$ & AKI $n=23$ & $p$ \\
\hline Age (years) & $47.1 \pm 14.5$ & $57.13 \pm 18.6$ & 0.01 \\
\hline Male sex (\%) & $46(64.8)$ & $17(74)$ & 0.42 \\
\hline Creatinine at admission (mg/dl) & $0.81 \pm 0.24$ & $1.09 \pm 0.24$ & 0.001 \\
\hline Higher Creatinine $(\mathrm{mg} / \mathrm{dl})$ & $0.98 \pm 0.6$ & $2.15 \pm 1.46$ & $<0.001$ \\
\hline Creatinine at discharge (mg/dl) & $0.74 \pm 0.47$ & $1.37 \pm 0.86$ & $<0.001$ \\
\hline \multicolumn{4}{|l|}{ Comorbidities (\%) } \\
\hline Hypertension & $20(28.2)$ & $15(65.2)$ & 0.001 \\
\hline Diabetes & $17(24)$ & $10(43.5)$ & 0.07 \\
\hline CKD & $4(5.6)$ & $1(4.3)$ & 0.81 \\
\hline Cardiovascular disease & $8(11.3)$ & $5(21.7)$ & 0.20 \\
\hline Cancer & $14(19.7)$ & $2(8.7)$ & 0.22 \\
\hline \multicolumn{4}{|l|}{ Cause of hospitalization (\%) } \\
\hline Infections & $16(22.5)$ & $12(50.0)$ & 0.02 \\
\hline Cardiovascular & $5(7)$ & 4 (16.6) & 0.20 \\
\hline Surgery & $38(53.5)$ & $5(20.4)$ & 0.04 \\
\hline Time of hospitalization (days) & $34.5 \pm 13.8$ & $33.6 \pm 10.94$ & 0.9 \\
\hline Time of vancomycin use (days) & $14.7 \pm 9.6$ & $17.3 \pm 7.04$ & 0.24 \\
\hline Vancomycin doses/day (g) & $2.5 \pm 0.9$ & $2.24 \pm 1.1$ & 0.48 \\
\hline Vancomycin concentration T0-48 h (mg/L) & $14.5 \pm 9.3$ & $18.3 \pm 15.1$ & 0.15 \\
\hline Vancomycin concentration T48-96 h (mg/L) & $15.4 \pm 8.9$ & $21.7 \pm 9.7$ & 0.006 \\
\hline Vancomycin concentration T96-144 h (mg/L) & $17.1 \pm 10.1$ & $24.2 \pm 19.1$ & 0.03 \\
\hline Vancomycin concentration T144-192 h (mg/L) & $16.5 \pm 9.1$ & $24.3 \pm 7.7$ & 0.003 \\
\hline Vancomycin concentration T192-240 (mg/L) & $17.5 \pm 9.6$ & $28.4 \pm 18.5$ & 0.016 \\
\hline Vancomycin concentration T240-288 h (mg/L) & $21.3 \pm 16.6$ & $32.4 \pm 27.1$ & 0.161 \\
\hline Death $(\%)$ & $6(8.4)$ & $2(8.7)$ & 0.97 \\
\hline
\end{tabular}

CKD, chronic kidney disease; T, time of vancomycin use.

TABLE 2 | Urinary biomarkers values according to the presence of acute kidney injury in patients hospitalized in wards and receiving vancomycin.

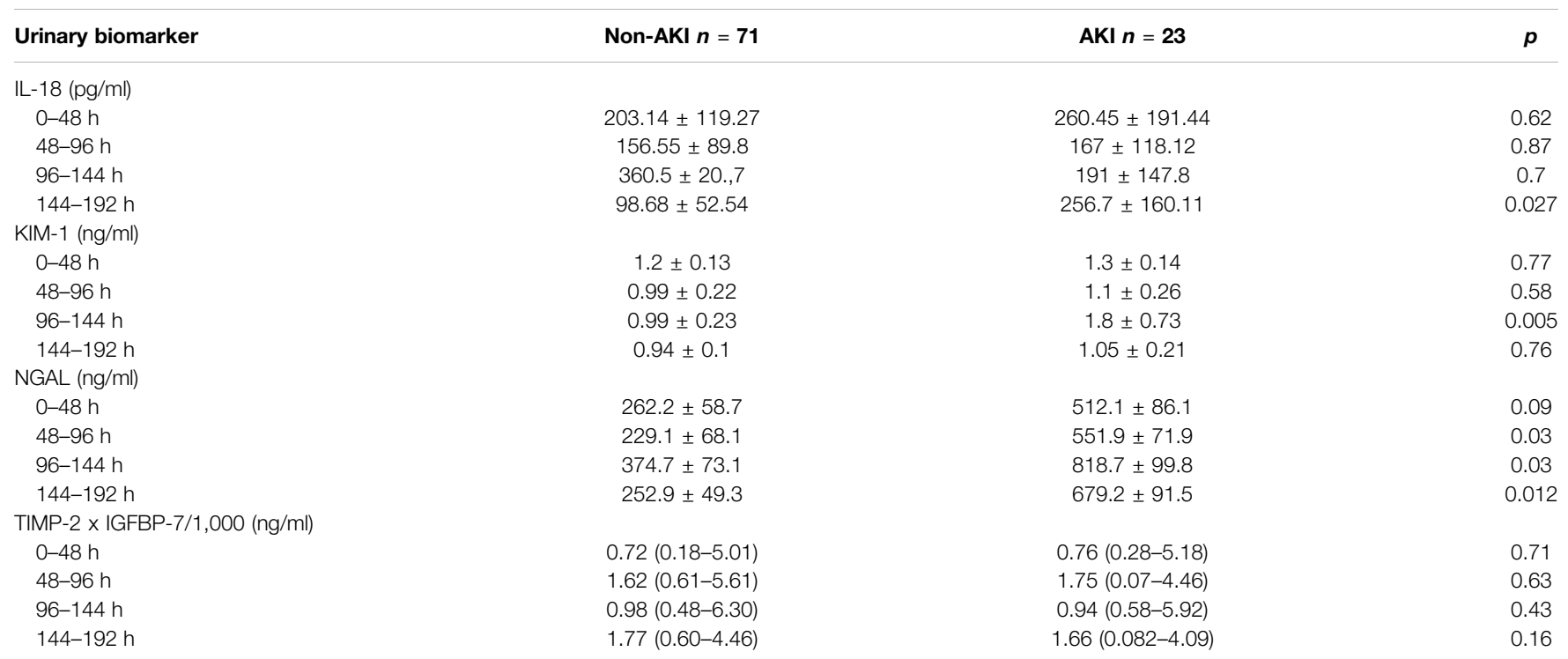

IL-18, interleukin 18; KIM-1, kidney injury molecule-1; NGAL, neutrophil gelatinase-associated lipocalin; TIMP-2, tissue inhibitor of metalloproteinases 2; IGFBP-7, insulin-like growth factor binding protein 7.

AKI patients were compared in relation to the presence or absence of kidney recovery at hospital discharge, and it occurred in 12 patients (52.2\%). The groups were similar in all the variables analyzed, except for the time of vancomycin use, which was longer in the group who did not recover kidney function $(21.1 \pm 7.6$ vs. $14.16 \pm 4.9$ days, $p=0.017)$, as shown in Table 5. The two groups were similar in urinary biomarkers, as shown in Table 6 and Table 7. Only urinary TIMP-2 concentration multiplied by the IGFBP-7 and normalized by urinary creatinine was higher between 144 and $192 \mathrm{~h}$ in 
TABLE 3 | Urinary biomarkers values normalized for urinary creatinine according to presence of acute kidney injury in patients hospitalized in wards and receiving vancomycin.

\begin{tabular}{|c|c|c|c|}
\hline $\begin{array}{l}\text { Urinary } \\
\text { biomarker/creatinine (mg/dl/mg/dl) }\end{array}$ & Non-AKI $n=71$ & AKI $n=23$ & $p$ \\
\hline \multicolumn{4}{|l|}{ IL-18 $\left(\times 10^{-7}\right) / \mathrm{cr}$} \\
\hline 1 (até 48 h) & $4.56 \pm 2.067$ & $7.12 \pm 1.28$ & 0.25 \\
\hline 2 (48-96 h) & $4.95 \pm 2.42$ & $5.34 \pm 1.81$ & 0.84 \\
\hline 3 (96-144 h) & $8.92 \pm 4.188$ & $5.46 \pm 2.43$ & 0.68 \\
\hline 4 (144-192 h) & $2.44 \pm 1.73$ & $10.18 \pm 6.56$ & 0.03 \\
\hline \multicolumn{4}{|l|}{$\mathrm{KIM}-1\left(\times 10^{-7}\right) / \mathrm{cr}$} \\
\hline 1 (até 48 h) & $33.68 \pm 7.90$ & $38.40 \pm 4.24$ & 0.62 \\
\hline 2 (48-96 h) & $37.30 \pm 6.94$ & $42.18 \pm 6.31$ & 0.74 \\
\hline 3 (96-144 h) & $37.08 \pm 6.49$ & $49.32 \pm 6.01$ & 0.33 \\
\hline 4 (144-192 h) & $29.24 \pm 8.98$ & $49.91 \pm 8.20$ & 0.29 \\
\hline \multicolumn{4}{|l|}{$\mathrm{NGAL}\left(\times 10^{-7}\right) / \mathrm{cr}$} \\
\hline 1 (até 48 h) & $69.484 \pm 13.19$ & $210.94 \pm 35.86$ & 0.05 \\
\hline 2 (48-96 h) & $181.67 \pm 63.84$ & $225.67 \pm 44.60$ & 0.008 \\
\hline 3 (96-144 h) & $191.28 \pm 50.19$ & $272.29 \pm 44.86$ & 0.55 \\
\hline 4 (144-192 h) & $36.18 \pm 9.31$ & $398.17 \pm 87.13$ & 0.05 \\
\hline \multicolumn{4}{|l|}{ TIMP-2 x IGFBP/cr } \\
\hline 1 (até 48 h) & $48.11 \pm 11.65$ & $43.11 \pm 29.13$ & 0.76 \\
\hline 2 (48-96 h) & $49.21 \pm 17.43$ & $53.14 \pm 31.42$ & 0.84 \\
\hline 3 (96-144 h) & $51.17 \pm 19.23$ & $66.76 \pm 41.41$ & 0.69 \\
\hline 4 (144-192 h) & $58.16 \pm 25.12$ & $282.52 \pm 66.25$ & 0.25 \\
\hline
\end{tabular}

IL-18, interleukin 18; KIM-1, kidney injury molecule-1; NGAL, neutrophil gelatinase-associated lipocalin; TIMP-2, tissue inhibitor of metalloproteinases 2; IGFBP-7, insulin-like growth factor binding protein 7

TABLE 4 | Logistic Regression associated with AKI.

\begin{tabular}{lcc}
\hline & Odds Ratio (95\% IC) & $\boldsymbol{p}$ \\
\hline Age & $6.66(1.16-38.2)$ & 0.03 \\
Vancomycin concentration (T 96-144 h) & $1.19(1.004-1.4)$ & 0.044 \\
Urinary NGAL (T 96-144 h) & $1.123(1.09-1.29)$ & 0.031
\end{tabular}

AKI, acute kidney injury; NGAL, neutrophil gelatinase-associated lipocalin; T, time of vancomycin use.

patients who did not recovery kidney function $(p=0.004$ and $p=0.03$, respectively).

Logistic regression identified non-recovery of kidney function after AKI, the time of vancomycin use (OR 4.6, CI 1.11-22.02, $p=0.03$ ), TIMP-2 multiplied by IGFBP -7 between 144 and $192 \mathrm{~h}$ normalized by urinary creatinine as predictors (OR 1.26, CI 1.092-1.543, $p=0.03$ ), as shown in Table 8.

The areas under the curve for vancomycin concentration and urinary NGAL levels between 96 and $144 \mathrm{~h}$ were 0.76 and 0.82 , respectively. Both plasmatic vancomycin concentrations and urNGAL were good predictors of AKI within the next 5 days. The optimal cutoff value of them had sensitivity and specificity of 0.79 and $0.66,0.83$ and 0.68, respectively (Table 9, Figure 2 and Figure 3).

Concerning urinary biomarkers of cell cycle arrest as predictors of recovery of kidney function at discharge hospital, the area under the curve for TIMP-2 multiplied by IGFBP-7 and normalized by urinary creatinine between 144 and $192 \mathrm{~h}$ was 0.71 , (Table 7, Figure 4). The optimal cutoff value was 0.3 , and sensibility and specificity were 0.88 and 0.64 .

\section{DISCUSSION}

AKI is a common and important condition to be recognized, depending on the serum creatinine for its diagnosis. Unfortunately, creatinine is a late and unreliable biomarker. According to Ostermann et al., there is a persistent unmet need for an earlier identification of patients with AKI. Furthermore, diagnostic tools that identify the location, mechanism, etiology, severity, and prognosis of AKI are necessary and have stimulated studies on new biomarkers, among them the IL-18, NGAL, KIM-1, TIMP-2, and IGFBP-7, identifying promising predictors of AKI in different scenarios such as cardiac surgery and sepsis (Wagener et al., 2006; Hirsch et al., 2007; Bagshaw et al., 2010; Liu et al., 2013; Singa et al., 2015; Luo et al., 2016; Mcduffie et al., 2016; Rocha et al., 2018; Ostermann et al., 2020); however, few studies evaluated the role of urinary biomarkers in AKI associated with vancomycin nephrotoxicity.

Despite the widespread use of vancomycin in practice, concerns related to its safety still permeate the academic environment. Emphasis has been given to its nephrotoxicity, with the risk of AKI with short- and longterm complications. Ninety-four patients were included while 114 were excluded, mainly because they already had AKI before using vancomycin (81) or because of the difficulty in urinary collection (Funk et al., 2016), which reflects the difficulty in studying the role of vancomycin nephrotoxicity, leading to doubt whether the high serum vancomycin concentrations are the cause or the consequence of AKI (Bosso et al., 2011; Elyasi et al., 2012; Hanrahan et al., 2014; Zamoner et al., 2020). 
TABLE 5 | Demographics and clinical characteristics of AKI patients according to the recovery of kidney function at hospital discharge.

\begin{tabular}{|c|c|c|c|}
\hline & $\begin{array}{l}\text { Non-kidney function recovery } \\
\qquad n=11\end{array}$ & $\begin{array}{l}\text { Recovery of kidney } \\
\text { function } n=12\end{array}$ & $p$ \\
\hline Age (years) & $62 \pm 19.5$ & $52.6 \pm 17.4$ & 0.23 \\
\hline Male sex (\%) & $9(82)$ & $8(67)$ & 0.40 \\
\hline Creatinine at admission (mg/dl) & $1.21 \pm 0.67$ & $0.98 \pm 0.34$ & 0.31 \\
\hline Higher creatinine (mg/dl) & $2.74 \pm 2.12$ & $1.6 \pm 0.6$ & 0.08 \\
\hline Creatinine at discharge (mg/dl) & $2 \pm 0.87$ & $0.8 \pm 0.19$ & $<0.001$ \\
\hline Comorbidities (\%) & & & 0.34 \\
\hline Hypertension & $6(54)$ & $9(75)$ & \\
\hline Diabetes & $4(36)$ & $6(50)$ & \\
\hline CKD & $1(9)$ & 0 & \\
\hline Cause of hospitalization (\%) & & & 0.36 \\
\hline Infections & $5(45)$ & $5(42)$ & \\
\hline Cardiovascular & $3(27)$ & $5(42)$ & \\
\hline KDIGO (\%) & & & 0.31 \\
\hline 1 & $5(45)$ & $9(75)$ & \\
\hline 2 & $3(27)$ & $1(8)$ & \\
\hline 3 & $3(27)$ & $2(16)$ & \\
\hline Time of hospitalization (days) & $34.4 \pm 12.19$ & $32.9 \pm 10.16$ & 0.76 \\
\hline Time of vancomycin use (days) & $21.1 \pm 7.6$ & $14.16 \pm 4.9$ & 0.017 \\
\hline Vancomycin doses/day (g) & $2.1 \pm 0.77$ & $2.39 \pm 1.33$ & 0.49 \\
\hline Vancomycin concentration T0-48 h (mg/L) & $24.7 \pm 19$ & $12.5 \pm 6.6$ & 0.47 \\
\hline Vancomycin concentration T48-96 h (mg/L) & $22.9 \pm 10.4$ & $20.5 \pm 9.2$ & 0.58 \\
\hline Vancomycin concentration T96-144 h (mg/L) & $25.1 \pm 22.7$ & $23.4 \pm 16$ & 0.84 \\
\hline Vancomycin concentration T144-192 h (mg/L) & $23.3 \pm 6.4$ & $25.75 \pm 9.56$ & 0.53 \\
\hline Vancomycin concentration T192-240 (mg/L) & $35.1 \pm 18.9$ & $15.37 \pm 10.7$ & 0.047 \\
\hline Death $(\%)$ & $2(18)$ & 0 & 0.12 \\
\hline
\end{tabular}

$C K D$, chronic kidney disease; $T$, time of vancomycin use.

TABLE 6 | Urinary biomarkers value according to the recovery of kidney function at discharge hospital in AKI patients hospitalized in wards and receiving vancomycin.

\begin{tabular}{|c|c|c|c|}
\hline Urinary biomarker & $\begin{array}{l}\text { Non-kidney function recovery } \\
\qquad n=11\end{array}$ & $\begin{array}{l}\text { Recovery of kidney } \\
\text { function } n=12\end{array}$ & $p$ \\
\hline \multicolumn{4}{|l|}{ IL-18 (pg/ml) } \\
\hline $0-48 \mathrm{~h}$ & $303.14 \pm 159.27$ & $210.45 \pm 191.44$ & 0.45 \\
\hline $48-96 \mathrm{~h}$ & $186.55 \pm 89.8$ & $152 \pm 58.12$ & 0.74 \\
\hline $96-144 \mathrm{~h}$ & $183.5 \pm 60.7$ & $198 \pm 37.8$ & 0.88 \\
\hline 144-192 h & $327.68 \pm 152.54$ & $205.7 \pm 60.11$ & 0.38 \\
\hline \multicolumn{4}{|l|}{ KIM-1 (ng/ml) } \\
\hline 0-48 h & $1.5 \pm 0.18$ & $1.11 \pm 0.74$ & 0.41 \\
\hline $48-96 \mathrm{~h}$ & $1.19 \pm 0.22$ & $1.08 \pm 0.26$ & 0.68 \\
\hline $96-144 \mathrm{~h}$ & $1.69 \pm 0.19$ & $1.92 \pm 0.83$ & 0.73 \\
\hline 144-192 h & $1.14 \pm 0.31$ & $1.06 \pm 0.31$ & 0.96 \\
\hline \multicolumn{4}{|l|}{ NGAL (ng/ml) } \\
\hline $0-48 \mathrm{~h}$ & $762.2 \pm 358.7$ & $512.1 \pm 86.1$ & 0.28 \\
\hline $48-96 \mathrm{~h}$ & $729.1 \pm 368.1$ & $751.9 \pm 271.9$ & 0.33 \\
\hline $96-144 \mathrm{~h}$ & $974.7 \pm 473.1$ & $918.7 \pm 399.8$ & 0.71 \\
\hline 144-192 h & $1,252.9 \pm 849.3$ & $679.2 \pm 291.5$ & 0.17 \\
\hline \multicolumn{4}{|c|}{ TIMP-2 x IGFBP-7/1,000 (ng/ml) } \\
\hline $0-48 \mathrm{~h}$ & $0.76(0.18-5.01)$ & $0.26(0.08-1.18)$ & 0.28 \\
\hline $48-96 \mathrm{~h}$ & $2.48(0.61-5.59)$ & $1.75(0.07-2.46)$ & 0.10 \\
\hline $96-144 \mathrm{~h}$ & 1.69 (0.88-6.30) & $1.11(0.78-3.92)$ & 0.37 \\
\hline 144-192 h & $2.76(1.60-4.46)$ & $1.06(0.08-2.09)$ & 0.05 \\
\hline
\end{tabular}

IL-18, interleukin 18; KIM-1, kidney injury molecule-1; NGAL, neutrophil gelatinase-associated lipocalin; TIMP-2, tissue inhibitor of metalloproteinases 2; IGFBP-7, insulin-like growth factor binding protein 7

Among the population studied, the incidence of AKI was 24.5\%, with a predominance of KDIGO $1(61 \%)$ and mortality of $8.7 \%$, which indicates that we really studied without AKI associated with sepsis, in which mortality is much higher, around 35\% (Srisawat and
Kellum, 2011). Data from the previous studies suggest variable nephrotoxicity prevalence, from 5 to $40 \%$ in the presence of other nephrotoxic drugs (Elyasi et al., 2012; Hanrahan et al., 2014; Pazhayattil and Shirali, 2014; Funk et al., 2016; Zamoner et al., 2020). 
TABLE 7 | Urinary biomarkers values normalized for urinary creatinine according to the recovery of kidney function at discharge hospital in patients hospitalized in wards and receiving vancomycin.

\begin{tabular}{|c|c|c|c|}
\hline $\begin{array}{l}\text { Urinary } \\
\text { biomarker/creatinine (mg/dl/mg/dl) }\end{array}$ & $\begin{array}{l}\text { Non-kidney function recovery } \\
\qquad n=11\end{array}$ & $\begin{array}{l}\text { Recovery of kidney } \\
\text { function } n=12\end{array}$ & $p$ \\
\hline \multicolumn{4}{|l|}{ IL-18 (x 10-7) } \\
\hline 1 (até 48 h) & $7.91 \pm 5.82$ & $6.40 \pm 1.06$ & 0.73 \\
\hline 2 (48-96 h) & $5.14 \pm 3.92$ & $5.51 \pm 3.03$ & 0.90 \\
\hline 3 (96-144 h) & $6.97 \pm 2.59$ & $4.08 \pm 3.43$ & 0.47 \\
\hline 4 (144-192 h) & $14.41 \pm 3.13$ & $6.55 \pm 2.35$ & 0.26 \\
\hline \multicolumn{4}{|l|}{$\mathrm{KIM}-1\left(\times 10^{-7}\right)$} \\
\hline 1 (até 48 h) & $32,28 \pm 4,80$ & $44.01 \pm 5.95$ & 0.56 \\
\hline 2 (48-96 h) & $43,98 \pm 6,70$ & $40.67 \pm 6.03$ & 0.90 \\
\hline 3 (96-144 h) & $55,65 \pm 8,12$ & $43.52 \pm 4.39$ & 0.65 \\
\hline 4 (144-192 h) & $60,24 \pm 16,89$ & $41.07 \pm 6.19$ & 0.46 \\
\hline \multicolumn{4}{|l|}{ NGAL $\left(\times 10^{-7}\right)$} \\
\hline 1 (até 48 h) & $263.68 \pm 45.49$ & $161.85 \pm 31.61$ & 0.51 \\
\hline 2 (48-96 h) & $306.70 \pm 56.65$ & $157.64 \pm 26.03$ & 0.43 \\
\hline 3 (96-144 h) & $398.52 \pm 55.79$ & $157.75 \pm 20.50$ & 0.20 \\
\hline 4 (144-192 h) & $684.27 \pm 126.86$ & $153.08 \pm 20.10$ & 0.68 \\
\hline \multicolumn{4}{|l|}{ TIMP-2 x IGFBP/cr } \\
\hline 1 (até 48 h) & $62.10 \pm 23.19$ & $56.12 \pm 9.49$ & 0.28 \\
\hline 2 (48-96 h) & $74.25 \pm 15.39$ & $65.23 \pm 25.19$ & 0.10 \\
\hline 3 (96-144 h) & $78.71 \pm 41.44$ & $51.91 \pm 16.84$ & 0.37 \\
\hline 4 (144-192 h) & $22.77 \pm 4.47$ & $10.66 \pm 4.44$ & 0.03 \\
\hline
\end{tabular}

IL-18, interleukin 18; KIM-1, kidney injury molecule-1; NGAL, neutrophil gelatinase-associated lipocalin; TIMP-2, tissue inhibitor of metalloproteinases 2; IGFBP-7, insulin-like growth factor binding protein 7

TABLE 8 | Logistic Regression associated with recovery of kidney function.

\begin{tabular}{lcc}
\hline & Odds Ratio (95\% IC) & $\boldsymbol{p}$ \\
\hline Time of vancomycin use (days) & $4.61(1.11-22.02)$ & 0.03 \\
UTIMP-2xIGFBP-7/cr (144-192 h) & $1.26(1.09-1.54)$ & 0.03
\end{tabular}

At logistic regression, only older age, higher creatinine at admission, and concentrations of vancomycin and urinary NGAL between 96 and $144 \mathrm{~h}$ were factors associated with AKI.

According to several studies, the age of patients remains as one of the most important risk factors for AKI, due to kidney senility, high frequency of comorbidities, and several medical procedures (Funk et al., 2016; Petronijevic et al., 2017).

Serum concentration $>23.8 \mathrm{mg} / \mathrm{L}$ and $\mathrm{u}$ NGAL $>618 \mathrm{ng} / \mathrm{ml}$ showed to be good predictors of AKI, with an area under the curve of 0.76 and 0.82 , the sensitivity of $79 \%$ and $83 \%$, the specificity of $66 \%$ and $68 \%$, respectively, and both of them preceded the diagnosis of AKI by 5 days (AKI happened on average on the eleventh day of vancomycin use).
Bosso et al. (Hirsch et al., 2007) performed a study that evaluated 288 patients using vancomycin and showed an association between nephrotoxicity incidence and vancomycin concentrations, with AKI occurring in almost $30 \%$ of patients with plasmatic concentrations of vancomycin $>15 \mathrm{mg} / \mathrm{L}$ and $9 \%$ of patients with vancomycin concentrations $<15 \mathrm{mg} / \mathrm{L}$. Recently, Zamoner et al. (2020) found that plasmatic concentration of vancomycin was an excellent predictor of AKI in patients admitted to wards, preceding the diagnosis of AKI by at least $72 \mathrm{~h}$.

The two urinary biomarkers studied most often for early diagnosis of AKI have been urine IL-18 and NGAL, mainly in sepsis and patients undergoing cardiac surgery. NGAL performed very well in two specific populations: children undergoing cardiac surgery and patients receiving kidney transplantation; however, it did not perform as well in critically ill children or adults and in adults undergoing cardiac surgery (Levey et al., 2009; Schiffl and Lang, 2012; Liu et al., 2013; Ronco, 2014).

The AUCs for IL-18 in four studies varied markedly (0.54-0.9). In general, IL-18 showed low sensitivity but

TABLE 9 | Sensitivity, specificity, area under curve, cutoff values of urinary biomarkers, and vancomycin concentration as predictors of diagnosis and recovery of kidney function in patients undergoing vancomycin and hospitalized in wards.

\begin{tabular}{|c|c|c|c|c|c|c|}
\hline & AUC & $p$-value & Cutoff & Sens & Spe & IC \\
\hline \multicolumn{7}{|l|}{ AKI diagnosis } \\
\hline Vancomycin concentration (T 96-144 h) & 0.76 & 0.03 & 23.8 & 0.69 & 0.66 & $0.71-0.98$ \\
\hline Urinary NGAL (T 96-144 h) & 0.82 & 0.02 & 618.8 & 0.73 & 0.68 & $0.61-0.96$ \\
\hline \multicolumn{7}{|l|}{ Recovery of kidney function } \\
\hline Urinary TIMP-2xIGFBP-7/1,000 (144-192 h) & 0.71 & 0.009 & 2.15 & 0.88 & 0.64 & $0.62-0.98$ \\
\hline
\end{tabular}

AUC, area under curve; sens, sensitivity; spe, specificity. 

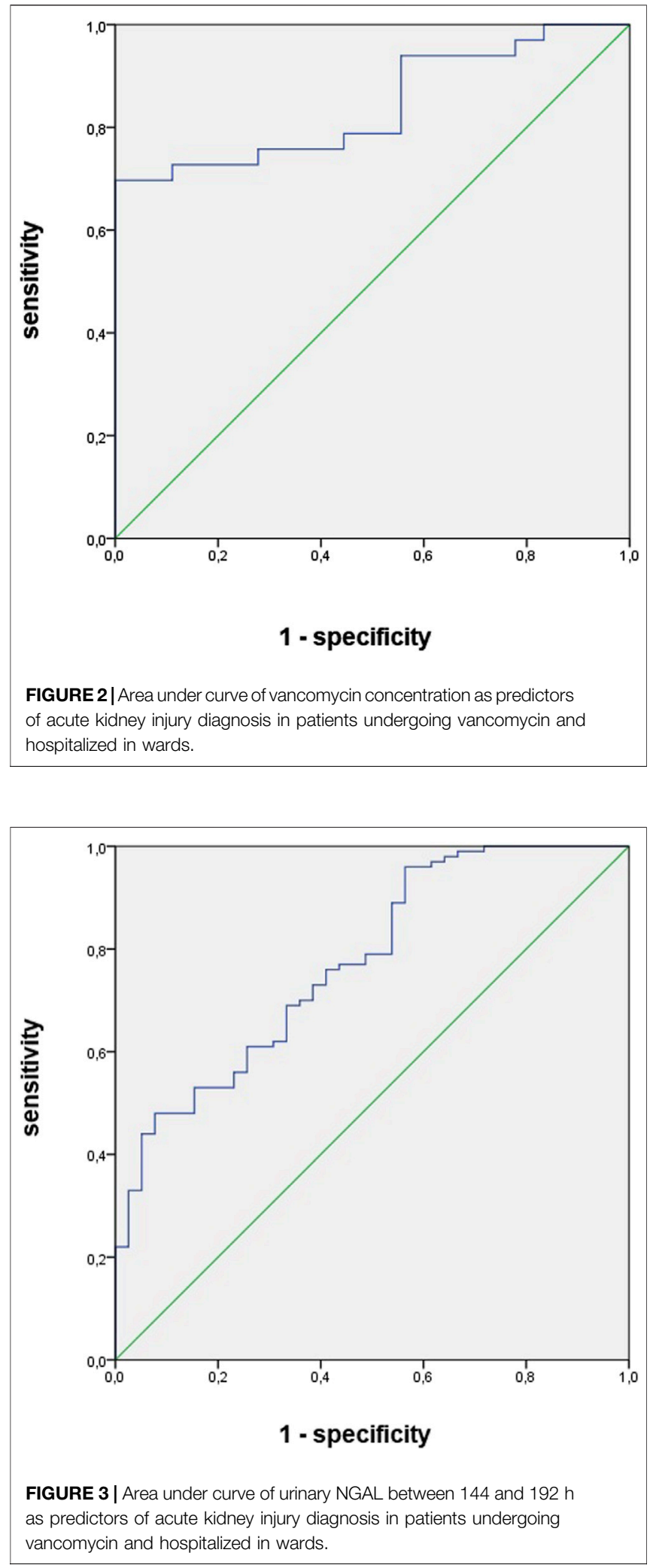

high specificity for the early diagnosis of AKI. Similar to NGAL, the performance of IL-18 is dependent on the time

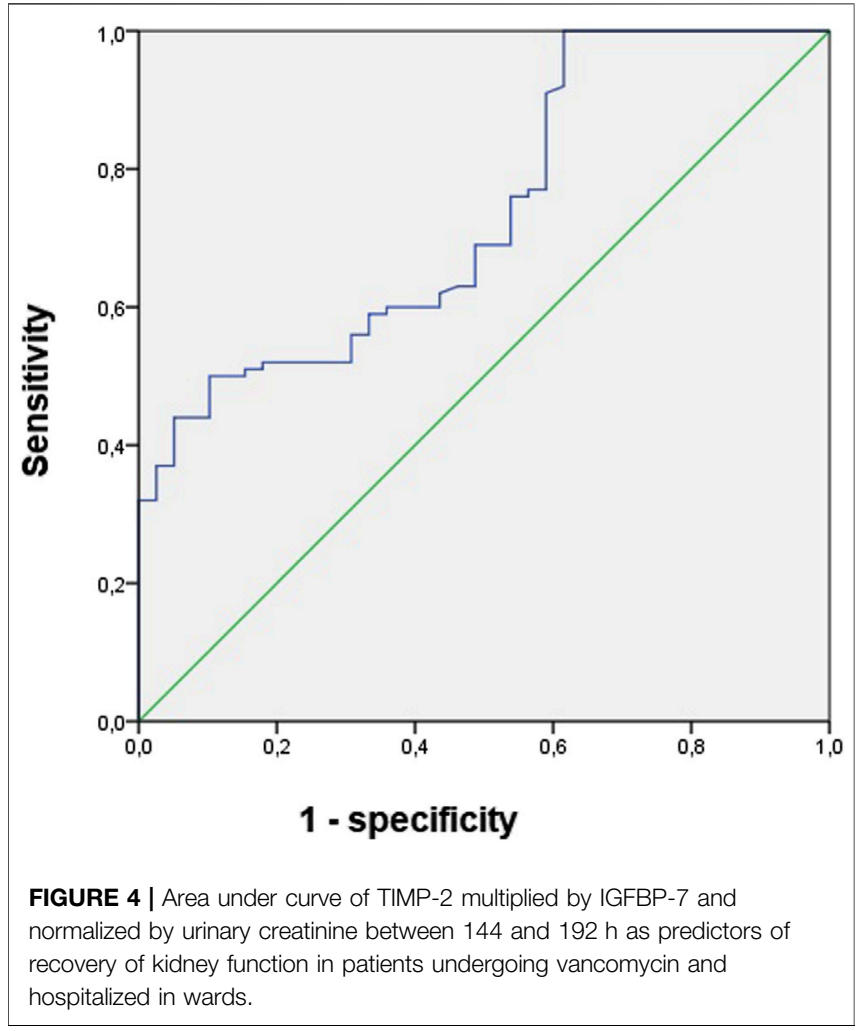

of collection in relation to the exposure (such as surgeries) and the AKI (Leslie and Meldrum, 2008). In summary, increases in urine IL-18 concentration are rarely false-positive elevations; however, several AKI patients do not have increased in urine IL-18.

Concerning KIM-1, it appears that it does not have good accuracy in early prediction of AKI, but it is good in identifying established AKI (Huang and Craig DonWauchope, 2011).

Regarding the non-recovery of renal function until the moment of hospital discharge, logistic regression identified the time of use of vancomycin and the highest TIMP-2 multiplied by IGFBP-7 between eighth and tenth days of antibiotic use as factors associated with non-recovery of renal function.

The area under the curve for TIMP-2 multiplied by IGFBP-7 was 0.71 , the optimal cutoff value was 2.35 , and sensibility and specificity were 0.88 and 0.64 .

To date, we are unaware of previous studies that have identified the length of use of vancomycin as a predictor of AKI prognosis.

Some biomarkers mentioned above were evaluated not only for AKI diagnosis but also for its prognosis, such as the recovery of kidney function.

TIMP-2 and IGFBP-7 have a direct role in cell cycle arrest mechanisms, and both proteins are expressed in tubular cells in response to DNA damage and possibly other forms of damage. The exact biological role of these proteins in AKI, in addition to their usefulness as biomarkers, is not fully understood (Ortega 
and Heung, 2018). Its levels are believed to decrease in the face of cell repair.

Ostermann et al. (2018), in an ancillary analysis of the multicenter SAPPHIRE study, examined the kinetics of the urinary TIMP-2 * IGFBP-7 in association with exposure to common renal insults (major surgery, IV radiocontrast, vancomycin, nonsteroidal anti-inflammatory, and piperacillin/ tazobactam). The authors compared the urinary TIMP-2 * IGFBP-7 kinetics from the day prior to exposure up to 5 days after exposure in patients developing AKI stage 2-3, stage 1 , or no AKI. Among the 723 patients, 679 (94\%) had at least one, 70\% had more than one, and $35 \%$ had three or more exposures to a known renal insult. There was a significant association between a cumulative number of exposures up to study day 3 and risk of AKI $(p=0.02)$ but no association between the specific type of exposure and AKI $(p=0.22)$. With the exception of radiocontrast, patients who developed AKI stage 2-3 after one of the five exposures had a clear rise and fall of urinary TIMP-2 * IGFBP-7.

The Sapphire study evaluated AKI prognosis, which includes death, need for dialysis, or persistence of renal dysfunction (creatinine above 2 times baseline) after 30 days (MAKE 30). The risk for MAKE 30 was lower when TIMP-2 • IGFBP7 <0.3 (18\%), increasing when TIMP-2 - IGFBP7 between 0.3 and $2(23 \%)$, and high when TIMP -2 • IGFBP7] 2 (40\%); $p<0.001$ (Kashani et al., 2013).

In this study, the biomarkers KIM-1 and IL-18 did not play a relevant role as diagnostic or prognostic predictors of AKI associated with vancomycin.

Our study presents some limitations. First, the sample obtained was small, reflecting the difficulty of studying nephrotoxicity. Second, the data were obtained from a single center. Third, there was absence of long-term follow-up, and finally, the role of plasmatic vancomycin concentration and urinary biomarkers was not studied as a prognostic predictor of AKI severity, of need for acute renal support, and of death due to the small number of events.

Despite these limitations, this is the first study to present cutoff of urinary biomarkers with the objective of evaluating their role as diagnostic and prognostic predictors of AKI associated with vancomycin.

\section{REFERENCES}

Andreucci, M., Faga, T., Pisani, A., Perticone, M., and Michael, A. (2017). The Ischemic/Nephrotoxic Acute Kidney Injury and the Use of Renal Biomarkers in Clinical Practice. Eur. J. Intern. Med. 39, 1-8. doi:10.1016/j.ejim.2016.12.001

Bagshaw, S. M., Bennett, M., Haase, M., Haase-Fielitz, A., Egi, M., Morimatsu, H., et al. (2010). Plasma and Urine Neutrophil Gelatinase-Associated Lipocalin in Septic versus Non-septic Acute Kidney Injury in Critical Illness. Intensive Care Med. 36 (3), 452-461. doi:10.1007/s00134-009-1724-9

Bosso, J. A., Nappi, J., Rudisill, C., Wellein, M., Bookstaver, P. B., Swindler, J., et al. (2011). Relationship Between Vancomycin Trough Concentrations and Nephrotoxicity: a Prospective Multicenter Trial. Antimicrob. Agents Chemother. 55 (12), 5475-5479. doi:10.1128/AAC.00168-11

Elyasi, S., Khalili, H., Dashti-Khavidaki, S., and Mohammadpour, A. (2012). Vancomycin-Induced Nephrotoxicity: Mechanism, Incidence, Risk Factors

\section{CONCLUSION}

Age, vancomycin concentrations, and urinary NGAL levels between 96 and $144 \mathrm{~h}$ are predictors of AKI, anticipating its diagnosis in at least 5 days, while the time of vancomycin use and urinary levels of TIMP-2. IGFBP-7 normalized by urinary creatinine between sixth and eighth day of vancomycin use were associated with TIMP-2 nonrecovery of kidney function among patients with AKI at the time of hospital discharge, indicating the possible evolution to chronic kidney disease. More and larger studies are needed to clarify the role of biomarkers in AKI associated with vancomycin.

\section{DATA AVAILABILITY STATEMENT}

The raw data supporting the conclusions of this article will be made available by the authors, without undue reservation.

\section{ETHICS STATEMENT}

The studies involving human participants were reviewed and approved by the Research Ethics Committee for analysis and started only after its approval (protocol 2,931,887). The patients/ participants provided their written informed consent to participate in this study.

\section{AUTHOR CONTRIBUTIONS}

Author contributions DS and AS collected data and prepared figures; DP and EB performed statistical analysis; LM and LA wrote the main manuscript text; DP, DS reviewed the final version of the study.

\section{SUPPLEMENTARY MATERIAL}

The Supplementary Material for this article can be found online at: https://www.frontiersin.org/articles/10.3389/fphar.2021.705636/ full\#supplementary-material

and Special Populations. A Literature Review. Eur. J. Clin. Pharmacol. 68, 1243-1255. doi:10.1007/s00228-012-1259-9

Funk, I., Seibert, E., Markau, S., and Girndt, M. (2016). Clinical Course of Acute Kidney Injury in Elderly Individuals Above 80 Years. Kidney Blood Press. Res. 41 (6), 947-955. doi:10.1159/000452599

Hanrahan, T. P., Harlow, G., Hutchinson, J., Dulhunty, J. M., Lipman, J., Whitehouse, T., et al. (2014). Vancomycin-Associated Nephrotoxicity in the Critically Ill. Crit. Care Med. 42 (12), 2527-2536. doi:10.1097/ CCM.0000000000000514

Hirsch, R., Dent, C., Pfriem, H., Allen, J., Beekman, R. H., Ma, Q., et al. (2007). NGAL Is an Early Predictive Biomarker of Contrast-Induced Nephropathy in Children. Pediatr. Nephrol. 22 (12), 2089-2095. doi:10.1007/s00467-0070601-4

Huang, Y., and Craig Don-Wauchope, A. (2011). The Clinical Utility of Kidney Injury Molecule 1 in the Prediction, Diagnosis and Prognosis of Acute Kidney Injury: a Systematic Review. Inflamm. Allergy Drug Targets. 10, 260-271. doi:10.2174/187152811796117735 
Kashani, K., Al-Khafaji, A., Ardiles, T., Artigas, A., Bagshaw, S. M., Bell, M., et al. (2013). Discovery and Validation of Cell Cycle Arrest Biomarkers in Human Acute Kidney Injury. Crit. Care. 17 (1), R25. doi:10.1186/cc12503

KDIGO Acute Kidney Injury Work Group (2012). KDIGO Clinical Practice Guideline for Acute Kidney Injury. Kidney Int. Suppl., 1-138. doi:10.1038/ kisup. 2012

Leslie, J. A., and Meldrum, K. K. (2008). The Role of Interleukin-18 in Renal Injury. J. Surg. Res. 145, 170-175. doi:10.1016/j.jss.2007.03.037

Levey, A. S., Stevens, L. A., Schmid, C. H., Zhang, Y., Castro, A. F., Feldman, H. I., et al. (2009). A New Equation to Estimate Glomerular Filtration Rate. Ann. Intern. Med. 150 (9), 604-612. doi:10.7326/0003-4819-150-9-200905050-00006

Liu, Y., Guo, W., Zhang, J., Xu, C., Yu, S., Mao, Z., et al. (2013). Urinary Interleukin 18 for Detection of Acute Kidney Injury: A Meta-Analysis. Am. J. Kidney Dis. 62 (6), 1058-1067. doi:10.1053/j.ajkd.2013.05.014

Luo, Q.-H., Chen, M.-L., Chen, Z.-L., Huang, C., Cheng, A.-C., Fang, J., et al. (2016). Evaluation of KIM-1 and NGAL as Early Indicators for Assessment of Gentamycin-Induced Nephrotoxicity In Vivo and In Vitro. Kidney Blood Press. Res. 41, 911-918. doi:10.1159/000452592

Mcduffie, J. E., Lee, S., Ma, J. Y., Chen, Y., and Snook, S. (2016). Acute Biomarker Panel Changes Associated with Amphotericin B Nephrotoxicity in Female Sprague-Dawley Rats. J. Toxicol. Sci. 41, 459-468. doi:10.2131/jts.41.459

Ortega, L. M., and Heung, M. (2018). The Use of Cell Cycle Arrest Biomarkers in the Early Detection of Acute Kidney Injury. Is This the New Renal Troponin? Nefrología (English Edition). 38, 355-361. doi:10.1016/j.nefro.2017.11.013

Ostermann, M., Mccullough, P. A., Forni, L. G., Bagshaw, S. M., Joannidis, M., Shi, J., et al. (2018). Kinetics of Urinary Cell Cycle Arrest Markers for Acute Kidney Injury Following Exposure to Potential Renal Insults. Crit. Care Med. 46, 375-383. doi:10.1097/CCM.0000000000002847

Ostermann, M., Zarbock, A., Goldstein, S., Kashani, K., Macedo, E., Murugan, R., et al. (2020). Recommendations on Acute Kidney Injury Biomarkers from the Acute Disease Quality Initiative Consensus Conference. JAMA Netw. Open. 3 (10), e2019209. doi:10.1001/jamanetworkopen.2020.19209

Pazhayattil, G. S., and Shirali, A. C. (2014). Drug-induced Impairment of Renal Function. Int. J. Nephrol. Renovasc Dis. 7, 457-468. doi:10.1055/s-0032-1305312

Petronijevic, Z., Selim, G., Petkovska, L., Georgievska-Ismail, L., Spasovski, G., and Tozija, L. (2017). The Effect of Treatment on Short-Term Outcomes in Elderly Patients with Acute Kidney Injury. Open Access Maced J. Med. Sci. 5 (5), 635-640. doi:10.3889/oamjms.2017.148

Rocha, E. P., Yokota, L. G., Sampaio, B. M., Eid, K. G. C., and Ponce, D. (2018). Urinary Neutrophil Gelatinase-Associated Lipocalin Is Excellent Predictor of Acute Kidney Injury in Septic Elderly Patients. Aging Dis. 9 (2), 182. doi:10.14336/AD.2017.0307

Ronco, C. (2014). Biomarkers for Acute Kidney Injury: Is NGAL Ready for Clinical Use? Crit. Care. 18, 680. doi:10.1186/s13054-014-0680-0

Rybak, M., Lomaestro, B., Rotschafer, J. C., Moellering, R., Craig, W., Billeter, M., et al. (2009). Therapeutic Monitoring of Vancomycin in Adult Patients: A
Consensus Review of the American Society of Health-System Pharmacists, the Infectious Diseases Society of America, and the Society of Infectious Diseases Pharmacists. Am. J. Health-System Pharm. 66, 82-98. doi:10.2146/ajhp080434 Schiffl, H., and Lang, S. M. (2012). Update on Biomarkers of Acute Kidney Injury. Mol. Diagn. Ther. 16, 199-207. doi:10.1007/BF03262209

Schrezenmeier, E. V., Barasch, J., Budde, K., Westhoff, T., and Schmidt-Ott, K. M. (2017). Biomarkers in Acute Kidney Injury - Pathophysiological Basis and Clinical Performance. Acta Physiol. 219 (3), 556-574. doi:10.1111/ apha. 12764

Siew, E. D., Matheny, M. E., Ikizler, T. A., Lewis, J. B., Miller, R. A., Waitman, L. R., et al. (2010). Commonly Used Surrogates for Baseline Renal Function Affect the Classification and Prognosis of Acute Kidney Injury. Kidney Int. 77, 536-542. doi:10.1038/ki.2009.479

Singa, H., Medeiros, P., Menezes, P., Bridi, R., Balbi, A., and Ponce, D. (2015). Sepsis and AKI in Clinical Emergency Room Patients: The Role of Urinary NGAL. Biomed. Res. Int. 2015, 1. doi:10.1155/2015/413751

Srisawat, N., and Kellum, J. A. (2011). Acute Kidney Injury. Curr. Opin. Crit. Care. 17 (6), 548-555. doi:10.1097/MCC.0b013e32834cd349

Wagener, G., Jan, M., Kim, M., Mori, K., Barasch, J. M., Sladen, R. N., et al. (2006). Association Between Increases in Urinary Neutrophil GelatinaseAssociated Lipocalin and Acute Renal Dysfunction after Adult Cardiac Surgery. Anesthesiology. 105 (3), 485-491. doi:10.1097/00000542200609000-00011

Zamoner, W., Gonçalves Pierri, I., Zanchetta Cardoso Eid, K., Maria Bellaver de Almeida, L., dos Santos, A., Luís Balbi, A., et al. (2020). Serum Concentration of Vancomycin Is a Diagnostic Predictor of Nephrotoxic Acute Kidney Injury in Septic Patients in Clinical and Surgical Wards. Infect. Drug Resist. 13, 403-411. doi:10.2147/IDR.S219989

Conflict of Interest: The authors declare that the research was conducted in the absence of any commercial or financial relationships that could be construed as a potential conflict of interest.

Publisher's Note: All claims expressed in this article are solely those of the authors and do not necessarily represent those of their affiliated organizations, or those of the publisher, the editors, and the reviewers. Any product that may be evaluated in this article, or claim that may be made by its manufacturer, is not guaranteed or endorsed by the publisher.

Copyright (c) 2021 Sampaio de Souza Garms, Cardoso Eid, Burdmann, Marçal, Antonângelo, Santos and Ponce. This is an open-access article distributed under the terms of the Creative Commons Attribution License (CC BY). The use, distribution or reproduction in other forums is permitted, provided the original author(s) and the copyright owner(s) are credited and that the original publication in this journal is cited, in accordance with accepted academic practice. No use, distribution or reproduction is permitted which does not comply with these terms. 